\title{
Trauma and orthopaedics in the COVID-19 pandemic: breaking every wave
}

Keng Jin Darren $\underline{T a y}^{1}$, MBBS, FRCS, Yee Han Dave $\underline{L e}^{2}$, MBBS, FRCS

\section{INTRODUCTION}

The coronavirus disease 2019 (COVID-19) pandemic has placed the whole world and the medical profession in an unprecedented situation. Each medical specialty has had to develop protocols within the local context to cope with this crisis. We aimed to provide a commentary on the evolving situation in orthopaedic departments in Singapore and share insights and recommendations for the consideration of orthopaedic surgeons worldwide.

The COVID-19 threat in Singapore can be classified into 'waves', which started with the confirmation of the first COVID19-positive patient in Singapore on 23 January 2020. The first wave of cases was traced to travel or contact with travellers to and from the Chinese city of Wuhan, Hubei. ${ }^{(1)}$ This progressed to more cases from North Asian cities in February 2020, with few cases from Europe or North America. As Singapore braced itself for community spread, the national disease threat level was stepped up from Disease Outbreak Response System Condition (DORSCON) Yellow to Orange on 7 February 2020..$^{(2-4)}$ By early March, the number of COVID-19-positive cases in Europe and North America had increased exponentially with a high number of fatalities, signalling a global pandemic.

The second wave of cases in Singapore, many of whom were returning students and Singaporeans working abroad, was traced to previous travel to Europe, North America and other cities in Asia. Unlike many countries that imposed a lockdown to limit local community spread, Singapore maintained a strategy of testing and isolation of infected individuals, and quarantine of all close contacts of infected individuals. This aggressive public health strategy allowed most Singaporeans to continue work and school with limited restrictions in February and March 2020. The policy of 'social distancing' was additionally promoted to mitigate local transmission.

The end of March 2020 saw multiple new local clusters and increased numbers of local unlinked COVID-19-positive cases. This heralded the start of the third wave of patients from significant local case transmission. ${ }^{(5)}$ Over this period, the resources of the local medical community - community practice, government restructured hospitals and the National Centre for Infectious Diseases (NCID) - were strained and further put to the test. The significant rise of new local unlinked cases necessitated a national 'circuit breaker' period that commenced on 7 April 2020 for one month (i.e. two COVID-19 incubation cycles). During this time, only essential services were permitted to continue. A nationwide school and non-essential workplace closure took effect in order to create a period of enhanced social distancing and reduced human traffic in Singapore.

Trauma and orthopaedics encompass both non-elective and elective work. Trauma involves the management of fractures that are categorised as urgent and semi-urgent. Elective orthopaedic surgery covers soft tissue repair and reconstruction, joint replacement in the shoulder, knee, ankle and hip joints, and spine surgery. Musculoskeletal oncology surgery and certain aspects of spine surgery such as spinal cord decompressions are generally considered urgent and semi-urgent. During this pandemic, orthopaedic departments in Singapore have to frequently calibrate and recalibrate their organisational structure and clinical operations as the crisis evolves.

\section{THE FIRST WAVE}

All hospitals in Singapore commenced visitation restrictions and stringent screening of admitted patients to ensure that those with a significant travel history to affected or high-risk regions were isolated. This allowed the rest of the hospital to function normally. All hospital staff were instructed to wear surgical masks in clinical areas. Additionally, in high-risk areas, such as the intensive care areas and emergency department resuscitation and fever areas, staff were provided with N95 masks. For aerosol-generating procedures, such as intubation, extubation and airway suctioning, the use of full personal protective equipment with N95 masks and protective eye shield was mandatory. To ensure the safety of healthcare workers (HCWs), mask fitting exercises and retraining on the use of powered air-purifying respirators (PAPRs) were carried out during this time. ${ }^{(6,7)}$

Management teams within orthopaedic departments began contingency planning to deal with the possible scenario of a HCW coming into contact with a COVID-19 patient, necessitating a 14-day quarantine. Larger departments with enough manpower planned for division into two or three functionally independent teams to ensure clinical work continuity. This was taken from Singapore's experience with the severe acute respiratory syndrome (SARS) outbreak in 2003, in which hospitals functioned with a 'clean' and 'dirty' team. The rotation and segregation of these teams allowed for an entire team ('dirty') to be quarantined should contact with an infected patient occur, and the other ('clean') to commence work. ${ }^{(8,9)}$

${ }^{1}$ Department of Orthopaedic Surgery, Singapore General Hospital, 2Department of Orthopaedic Surgery, Changi General Hospital, Singapore 


\section{DORSCON ORANGE AND THE SECOND WAVE}

As the DORSCON level escalated from Yellow to Orange, all orthopaedic departments curtailed elective surgeries. The guiding principle was based on preservation of inpatient beds and highdependency and intensive care resources, as well as limiting exposure of elective patients to a hospital stay and, potentially, an undiagnosed COVID-19 patient.

Day surgery procedures continued to be performed in most orthopaedic departments, and patients were discharged home following their procedures. Arthroscopic surgeries including knee, shoulder and ankle repairs and reconstruction were performed, along with day surgery trauma cases. Additionally, certain departments were further able to provide 23-hour-stay unicompartmental and total knee replacements, knee osteotomies and hip replacements without requiring inpatient admission during these two months. Doing so helped to limit the waiting time and build-up of elective cases.

Anaesthesia departments mandated splitting of medical manpower, which meant a reduction in operating theatres (OTs) for elective orthopaedic surgery. In addition, fewer cases could be operated on per surgical list, as additional time had to be factored in for longer and safer turnovers between patients. As a precautionary measure, all intubation and extubation procedures were to take place with only the anaesthesia team inside the OT to limit possible exposure of other HCWs to an undiagnosed COVID-19 patient. $^{(10,11)}$

Most departments experienced a significant drop in elective surgery workload. For example, only 20\% (February 2020) and $45 \%$ (March 2020) of planned electives took place in one orthopaedic department. This represented a drop of $55 \%-80 \%$ of orthopaedic elective surgeries during those two months. The decreased surgical workload in orthopaedic departments allowed medical and nursing manpower to be redeployed to other clinical areas that experienced a surge in manpower requirements, such as emergency departments, respiratory wards, isolation wards and the NCID.

On the average, each orthopaedic department sees 300-500 osteoporotic hip fractures annually, which translates to 5-10 hip fractures managed per week. Given the vulnerability of this patient population, higher priority for early surgery was given to these patients in order to minimise hospital stay, thereby freeing up inpatient beds and limiting the risk of in-hospital COVID-19 infection among elderly patients. This was similarly practised for all other fracture and trauma cases admitted through the emergency departments. In most departments, at least one dedicated OT was reserved solely for trauma surgery. This was staffed with a consultant-grade orthopaedic surgeon to deal expediently with hip, spine and other fractures to shorten operative times and minimise postoperative complications. Similar priority was given to other emergent and semi-emergent cases, such as those involving musculoskeletal oncology and spinal cord compression with deteriorating neurological status. During the months of February and March 2020, approximately 120-160 fracture repairs, wound debridement procedures and spine surgeries were performed each month in the hospital orthopaedic department.

\section{THE THIRD WAVE AND CIRCUIT BREAKER}

With the implementation of the circuit breaker on 7 April 2020, all non-urgent and non-time-critical elective procedures were cancelled. These included day cases, even procedures that were to be done under local anaesthesia. This was in line with the regulation of allowing only essential services during this period to ensure that people stay at home and reduce their exposure risk. Only surgeries such as fracture fixation, infection control, spinal trauma, cord compression and some musculoskeletal tumour resections were permitted to proceed on the basis that these patients would have adverse outcomes with threat to life or limb if the surgery were to be delayed or postponed.

In this third wave, when there is significant local community spread and rising numbers despite appropriate social distancing measures, precise planning is required to ensure that essential orthopaedic surgeries take place safely for orthopaedic surgeons and their team. In addition, non-urgent outpatient hospital visits have been postponed and measures to limit nonessential footfall in the hospitals (i.e. hospital distancing) have also been implemented. During this period, we provide some recommendations to orthopaedic surgeons for their consideration:

- Practise increased distancing and segregation between HCWs. In a report by Guo et al, it was suggested that where COVID-19 was endemic, the incidence of infection from other HCWs was reported to be $1.5 \%-20.7 \%$. The suspected sites of exposure were the general wards $(79.2 \%)$, hospital general areas $(20.8 \%)$, operating room $(12.5 \%)$, intensive care units $(4.2 \%)$ and clinics $(4.2 \%) .{ }^{(12)}$

- Implement screening of patients' COVID-19 status prior to orthopaedic surgery. Guo et al suggested that in endemic areas, patients should be screened for COVID-19 prior to surgery. This takes into account that up to $20 \%$ of people may be asymptomatic carriers and spreaders of COVID-19. Knowledge of a patient's COVID-19 status preoperatively allows the institution to put in place full protective measures for safe surgery. ${ }^{(11,12)}$

- Make preparations to operate on COVID-19 patients. Teams of anaesthetists, orthopaedic surgeons and scrub nurses should undergo training and simulations, and be familiar with the setup of the OT, the use of PAPRs for surgery, and the donning and doffing process. ${ }^{(13)}$

- Consider non-operative management of orthopaedic fractures where possible and appropriate (e.g. treat distal radius fractures with a splint). If conservative management is not possible, then consider delaying surgery to a later stage. ${ }^{(10,11)}$ Based on a study by Mi et al, fracture patients with COVID-19 infection have a poorer prognosis. In their series of ten patients with fractures and COVID infection, four patients died; one died 11 days after surgery, while the other three had no surgery performed. Three other cases had severe pneumonia. ${ }^{(14)}$ 
- $\quad$ Reduce inpatient hospital stays for elderly patients as much as possible. This is because elderly patients with lower limb fractures (e.g. hip fractures) in COVID-19-endemic areas are especially vulnerable to COVID-19 infection and at the highest risk of developing complications. Therefore, high standards of infection control are essential to reduce this risk. ${ }^{(14)}$

- $\quad$ Perform surgery for COVID-19 patients who require fracture fixation surgery in a negative pressure OT. It is suggested that everyone involved in the procedure be properly protected with PAPR. ${ }^{(10,11,13)}$ PAPRs draw ambient air through a highefficiency particulate air filter and their efficiency is superior to surgical helmets used by arthroplasty surgeons, as these hoods do not filter sub-micrometre particles. The use of power tools such as electrocautery, bone saws, drills and reamers generates aerosol, increasing the risk of infection, and should be minimised if possible. ${ }^{(15)}$

- Increase orthopaedic presence in emergency departments to ensure proper admission and right-siting of patients to the appropriate hospital service. This serves to protect the number of inpatient beds, limit unnecessary admissions and, in turn, reduce hospital exposure for the vulnerable elderly (i.e. hospital distancing for elderly patients). This also provides an opportunity for appropriate siting of patients to fracture clinics for early surgery without the need for an inpatient stay. ${ }^{(10)}$

- $\quad$ Consider increasing the use of teleconsultation for outpatient consultation. In place of postponing all non-urgent followup appointments, teleconsultations can be arranged for patients to update their surgeon on their current condition. This can help surgeons to determine when to reschedule the next follow-up. Teleconsultation can also be considered for some new outpatient appointments. Patient history and review of imaging (if already performed) can be done remotely to determine the next best plan. Again, this helps to establish the urgency level of the next outpatient clinic visit, instead of delaying the initial consultation. ${ }^{(10)}$

- Use wearable devices and telemedicine to reduce outpatient visits. This gives surgeons and physiotherapists the opportunity to track patient activity or range of joint motion via the wearable devices. The data can then be uploaded for review by the hospital staff. The use of such technology can complement patient rehabilitation, as compliance to home physiotherapy exercises can be monitored.

In conclusion, the COVID-19 pandemic requires the local orthopaedic community to join in the fight alongside Singapore. Social distancing, infection control, nimble recalibrations, resource optimisation, continuous innovation and selfless leadership are all essential as we prepare for a long-drawn battle.

\section{REFERENCES}

1. Huang C, Wang Y, Li X, et al. Clinical features of patients infected with 2019 novel coronavirus in Wuhan, China. Lancet 2020; 395:497-506.

2. Ministry of Health, Singapore. Updates on COVID-19 (coronavirus disease 2019) local situation. Available at: https://www.moh.gov.sg/covid-19. Accessed April 2, 2020.

3. Wong JEL, Leo YS, Tan CC. COVID-19 in Singapore-current experience: critical global issues that require attention and action. JAMA 2020 Feb 20. https://doi. org/10.1001/jama.2020.2467. [Epub ahead of print]

4. Young BE, Ong SWX, Kalimuddin S, et al. Epidemiologic features and clinical course of patients infected with SARS-CoV-2 in Singapore. JAMA 2020; 323:1488-94.

5. Wei WE, Li ZB, Chiew CJ, et al. Presymptomatic transmission of SARS-CoV-2-Singapore, January 23-March 16, 2020. MMWR Morb Mortal Wkly Rep 2020; 69:411-5.

6. $\mathrm{Ng} \mathrm{KQ}$, Poon BH, Puar HKT, et al. COVID-19 and the risk to health care workers: a case report. Ann Intern Med 2020; 172:766-7.

7. Ong SWX, Tan YK, Chia PY, et al. Air, surface environmental, and personal protective equipment contamination by severe acute respiratory syndrome coronavirus 2 (SARS-CoV-2) from a symptomatic patient. JAMA 2020; 323:1610-2.

8. Chang Liang Z, Wang W, Murphy D, Po Hui JH. Novel coronavirus and orthopaedic surgery early experiences from Singapore. J Bone Joint Surg Am 2020; 102:745-9.

9. Vannabouathong C, Devji T, Ekhtiari S, et al. Novel coronavirus COVID-19: current evidence and evolving strategies. J Bone Joint Surg Am 2020; 102:734-44.

10. The National Health Service, UK. Clinical guide for the management of trauma and orthopaedic patients during the coronavirus pandemic. Specialty guides for patient management during the coronavirus pandemic. Publications approval reference: 001559. 16 March 2020. Available at: https://www.england.nhs.uk/ coronavirus/wp-content/uploads/sites/52/2020/03/specialty-guide-orthopaedictrauma-and-coronavirus-v1-16-march-2020.pdf. Accessed April 3, 2020.

11. Royal Australasian College of Surgeons. RACS guidelines for the management of surgical patients during the COVID-19 pandemic. Available at: https:// umbraco.surgeons.org/media/5137/racs-guidelines-for-the-management-ofsurgical-patients-during-the-covid-19-pandemic.pdf. Accessed April 4, 2020.

12. Guo X, Wang J, Hu D, et al. Survey of COVID-19 disease among orthopaedic surgeons in Wuhan, People's Republic of China. J Bone Joint Surg Am 2020; 102:847-54

13. Rodrigues-Pinto R, Sousa R, Oliveira A. Preparing to perform trauma and orthopaedic surgery on patients with COVID-19. J Bone Joint Surg Am 2020; 102:946-50.

14. Mi B, Chen L, Xiong Y, Xue H, et al. Characteristics and early prognosis of COVID-19 infection in fracture patients. J Bone Joint Surg Am 2020; 102:750-8.

15. Yeh HC, Turner RS, Jones RK, et al. Characterization of aerosols produced during surgical procedures in hospitals. Aerosol Sci Technol 1995; 22:151-61. 